behandelt wird, während die größten Gruppen von 200 und 300 Personen mit keiner Zeile erwähnt werden. Es ist bedauerlich, daß in einem für die Information bestimmten Werk ein derart schiefes Bild entworfen wird. Auch die völlig falsche Angabe, daß der tibetische Kandschur eine Übersetzung des Pālikanons darstelle (S. 195), berührt etwas unangenehm.

Manchmal wäre eine noch bessere Abstimmung der Beiträge aufeinander wünschenswert. So weichen z. B. die Angaben über den Gründer der Maha-BodhiSociety in Schreibweise und Inhalt sehr voneinander ab: Anagārika Dharmapāla (David Hewavitarane), gestorben 1944 (S. 64) und Anagarika Dharmapala (David Hewawitarne) gestorben 1933 (S.72). Die richtige Angabe ist: Anagārika Dharmapāla (David Hewavitarne) gestorben 1933.

Dagegen sind trotz der vielen fremdsprachlichen Angaben Druckfehler selten, z. B. Surcey statt Survey (S. 56, Anm. 38) und Faktion statt Fraktion (S. 63).

H. Hecker

\section{Nagendra Singh}

The Theory of Force and Organisation of Defence in Indian Constitutional History, From earliest times to 1947 Asia Publishing House, London, 1969

Im März und im Mai dieses Jahres erschreckte der ehemalige Oberkommandierende der indischen Armee General K. M. Cariappa die indischen Parlamentarier mit dem Vorschlag, der Präsident solle gestützt auf die Armee die Macht übernehmen.

Da die parlamentarische Demokratie zur Zeit nicht in der Lage sei, Ruhe und Ordnung zu garantieren, und die Verfassung eine presidents rule wie die der Staaten für die Zentralregierung nicht vorsehe, müsse sie außer Kraft gesetzt werden. Allerdings lehnte der General in weiser Einschätzung der Stärke und Homogenität der Armee die Errichtung einer echten Militärdiktatur ab. Die Tatsache, daß der ehemalige Oberkommandierende der indischen Armee diese zur Einführung einer nicht verfassungsmä-
Bigen Ordnung benutzen will, ist deshalb so erstaunlich, weil sie in krassem Gegensatz zu der traditionellen Abstinenz der indischen Armee in politischen Fragen steht. Dies gibt Anlaß, auf das angezeigte Buch aufmerksam zu machen. Angesichts der wissenschaftlichen Qualifikation des Verfassers ist man nicht erstaunt, eine Arbeit vorzufinden, die sich nicht nur durch Gründlichkeit, sondern auch durch einen weiten Horizont auszeichnet. Zwar beschränkt sich der Verfasser auf die Darstellung der Verzahnung von Herrschaft und bewaffneter Macht, gibt aber doch einen Überblick über die Entwicklung der Verfassungen in Indien von den frühesten Zeiten bis zur Erringung der Unabhängigkeit.

Es ist erstaunlich, daß diese Fragen in der neueren Indienliteratur kaum besprochen worden sind. Dieses Buch schließt eine Lücke. Für den Juristen sind die Kapitel über das politische Denken in den jeweiligen Epochen besonders interessant, da sie Einblick in das Staatsdenken geben und damit zur Frage der Legitimierung von Herrschaft und Gewalt beitragen. Wer das Buch gelesen hat, wird mit dem Verfasser sagen können, daß in Indien eine der abendländischen durchaus ebenbürtige Entwicklung der Theorie von Staat und Macht, Herrschaft und Gewalt stattgefunden hat.

Das Buch ist so von hohem theoretischen und historischem Interesse. Das Problem, wie ein Staat die von ihm geschaffene Armee in ihren Schranken hält, muß jedoch immer wieder neu gelöst werden. Das angezeigte Buch kann Grundlage für eine Beschäftigung mit dieser in Indien wieder hoch aktuell gewordenen Frage sein.

Henning v. Wede!

\section{Eugene R. Black}

Alternative in Southeast Asia

London: Pall Mall Press 1969

$\mathrm{XV} ; 180$ S., £ 2.50

Eugene R. Black leitete von 1949 bis 1963 die Internationale Bank für Wiederaufbau und Entwicklung. In seinem ersten Buch mit dem Titel "Die Diplo- 
matie wirtschaftlicher Entwicklung" bereits bezeichnete er die Tätigkeit des Entwicklungsbankiers, wie er sich selbst nennt, nämlich reiche und arme Länder zusammenzubringen, als eine Kunst. 1965 berief Präsident Johnson ihn zu seinem Sonderberater, der für die Zeit nach der militärischen Intervention die Förderung der wirtschaftlichen Integration Südostasiens vorausdenken sollte. Das neue Buch von Black, der im Alter von 70 Jahren Direktorenstellungen der Chase Manhattan Bank, American Express Company, Royal Dutch Petroleum Company und der New York Times vereinigt, gibt nun Aufschluß über die Kunstmittel des Entwicklungsbankiers zur Gestaltung der zukünftigen Südostasienpolitik.

Es ist nicht anders zu erwarten, als daß Black für die Wiederherstellung des Friedens in Südostasien die Glaubwürdigkeit der Wirksamkeit der amerikanischen Militärmacht und der Entschlossenheit der US-Regierung voraussetzt, mit dieser Macht das nationale Interesse zu sichern (S. 12). Obwohl sich die amerikanischen Interessen in Südostasien z. B. nach den Kriterien Sicherheit, Profite, historische Verantwortung, Vertragstreue und regionale Stabilität von Staat zu Staat ziemlich differenziert analysieren lassen ${ }^{1}$, redefiniert Black pauschal als das amerikanische Interesse, die Beherrschung Asiens durch eine Einzelmacht, die Beherrschung Südostasiens durch China, zu verhüten (S. 8). Es erfrischt jedoch, wie Black die bisherige amerikanische 'Kombination von Entwicklungsprogrammen plus Counterinsurgency kritisiert (S. $16 \mathrm{f}$.):

$\mathrm{Da}$ die Counterinsurgency-Doktrin fälschlich von der Annahme ausging, daß die USA und die mit ihr kooperierenden Regierungen den Radikalisten selbst progressive Programme entgegensetzten, beanspruchte sie zu Unrecht, die USA könnten kommunistisch geführte nationale Befreiungskriege niederschlagen. Counterinsurgency ließ sich auch mit Entwicklungsprogrammen nicht rechtfertigen, weil es sich als Täuschung erwies, Entwicklung mit wirtschaftlichem Wachstum gleichzusetzen. Black empfiehlt als Alternative zur übermächtigen Selbstdarstellung der Amerikaner in Südostasien, einen multilateralen Rahmen für die Außenpolitik zu schaffen.

$\mathrm{Da}$ es Black also weniger auf eine als Welt-Innenpolitik $\mathrm{zu}$ verstehende Außenpolitik als auf die Befreiung Amerikas vom Vietnam-Komplex (S. 178) ankommt, beschränkt sich seine Bestandsaufnahme der Ansätze eines südostasiatischen Regionalismus auf ein paar Anekdoten. Hierauf bezog sich wohl der Kritiker der Far Eastern Economic Review $^{2}$, als er Blacks überflüssige Diskretion bemängelte und den gegenwärtigen Direktor der Weltbank, McNamara, aufforderte, nach seiner Amtszeit einen interessanteren Tätigkeitsbericht vorzulegen. Andererseits schlägt Black eine Reihe von Reformen vor, die die Wirksamkeit einer zeitgemäßen amerikanischen Außenpolitik gewährleisten sollen:

Indem Amerika bei der Gestaltung der Auslandshilfe anstatt Entwicklung, Wirtschaftswachstum förderte, habe es zuwenig auf Qualität und zuviel auf Quantität geachtet (S. 165). Zu einer Zeit, da keine Nation mehr bilateral auf sich Druck ausüben lasse, bleibe Entwicklungshilfe nur noch ein Mittel, den Kurs regionaler Institutionen zu beeinflussen (S. 90). Die neu gegründete Asiatische Entwicklungsbank und eine Mekong-Behörde, die zur Nutzbarmachung des $\mathrm{Me}$ kong von den Anliegerstaaten zu gründen sei, eigneten sich als Planungs- und Koordinationszentren. $\mathrm{Da}$ es vom Verhalten der anderen, nicht vom amerikanischen Verhalten abhänge, ob regionale Kooperation in Südostasien ein nützliches Ziel sei, müsse die Kunst der Diplomatie kultiviert werden (S. 90). Die amerikanische Diplomatie dürfe nicht länger Politikern, Militärs und anderen Amateuren überlassen bleiben, sondern ausschließlich Spezialisten im Dienst des State Department (S. 20-25). Regiona-

\footnotetext{
1 Gurtov, Melvin: Problems and Prospects of United States Policy in Southeast Asia. Santa Monica, Calif.: Rand Memorandum RM - 5910 - ISA 1969, S. 39 ff.).

269,28 vom 9. 7. 1970, S. 78.
} 
le Zusammenarbeit biete eine der ganz wenigen Gelegenheiten, einen Interessenkonflikt mit Japan zu vermeiden, im Gegenteil, Japan zu einem nützlichen Engagement herauszufordern (S. 67 bis 89). Für die zukünftigen bilateralen Programme solle ein Fonds mit 3 Billionen $\$$ Anfangskapital eingerichtet werden, so daß der Kongreß nur noch die Verwaltung dieses Fonds zu kontrollieren brauche (S. 70). Als Ausgleich zum Disengagement der zahlreichen offiziellen Kontaktstellen könnten die USA mehr mit den Universitäten, im Geschäftsleben und mit privaten Stiftungen gegenwärtig bleiben.

Klaus v. d. Decken

\section{J. P. W. B. McAuslan}

\section{The Evolution of Public Law in East} Africa in the $1960 \mathrm{~s}$

in: Public Law, Jahrgang 1970, S. 5 ff., S. $153 \mathrm{ff}$. (London-Stevens)

Diese Aufsatzfolge enthält so zahlreiche gute Beobachtungen und treffende Bemerkungen, daß es nicht leicht fällt, den roten Faden zu entdecken, der die Darstellung durchzieht - zumal es dem Verfasser darauf ankommt, gerade auch die Verschiedenheiten in der Verfassungsentwicklung der drei Staaten hervortreten $\mathrm{zu}$ lassen, auf die er seinen Blick beschränkt: Kenia, Tansania und Uganda. Ausgangspunkt ist die Konstellation im Augenblick der Erlangung der Selbständigkeit. Verfassungsrechtlicher Kern derselben ist eine überaus starke Exekutive, wie sie von der Politik der Kolonialherren gefordert war (zur Stellung der Rechtspflege in der Kolonialzeit vgl. S. 164 f). Gegenüber einer solchen Situation hätte "Befreiung“ per definitionem Entmachtung der Exekutive und Stärkung der beiden anderen Gewalten vor allem zwecks Verhinderung von Machtmißbrauchs der ersten verlangt. Dieses Ziel hatten sich denn auch folgerichtig die mit den Kolonialherren vereinbarten Verfassungen gesetzt, ja dieses Ziel in einzelnen Fällen bis zu einem solchen Extrem verwirklicht, daß das Übermaß der Rechtsstaatlichkeit selbst ein Mindestmaß von Effizienz ausschloß (S. 7; 16/17). Genau das Gegenteil aber war das Gebot der Stunde. Da Modernisierung ein Höchstmaß an Handlungsund Leistungsfähigkeit des Staates voraussetzt, ergibt sich die Richtung von selbst, die die Verfassungsentwicklung nach der Befreiung eingeschlagen hat: Sie entfernt sich alsbald von den vereinbarten Verfassungen und dem ihnen zugrundeliegenden „Westminster-Modell“ und gipfelt in einer praktischen Alleinherrschaft der Exekutive - eine Ähnlichkeit mit der Kolonialzeit, die immer wieder in der Wendung zum Ausdruck kommt, die Bevölkerung habe nicht die Herrschaft, sondern lediglich die Herren gewechselt.

Im zweiten Teil führt der Verfasser eine Auswahl der Institute und Prozeduren vor, aus denen diese Stärke der Exekutive erwächst (S. 158 ff.). An erster Stelle nennt er das Notrecht, dessen wirksamste Waffe der administrative Freiheitsentzug vor allem insofern ist als er die Ausschaltung von Opposition und Kritik ermöglicht. Aber auch ohnedem stehen der vollziehenden Gewalt zahlreiche und wirksame Mittel der Manipulation von Gesetzgebung und Rechtspflege zur Verfügung, einschließlich der Möglichkeit, die eine dieser Gewalten gegen die andere einzusetzen, etwa ein unerwünschtes Urteil durch ein gezieltes Gesetz aus der Welt zu schaffen (S. 166 ff.). Abhilfe gegen Mißbrauch ist schwach entwickelt, wenn man von Tansanias, sich am Ombudsman orientierender "Permanent Commission of Inquiry" (S. 168 ff.) absieht. Entscheidung für den Vorrang der Exekutive ist jedoch auch hier der Zwang der Sache selbst. Die Herstellung staatlicher Einheit aus und in einer zersplitterten, verschiedenartigen und verfeindeten Gesellschaft und die Ausstattung dieser Einheit mit derjenigen Handlungs- und Leistungsfähigkeit, wie sie von einer radikalen und schnellen Modernisierung gefordert werden, läßt offenbar einstweilen keine andere Lösung zu als die Konzentration der Macht in der Regierung (S. 159). Nur nebenbei bemerkt sei, daß in einer solchen Situation einer Verfassung die 